CAHIERS DE

NARRATOLOGIE

\section{Cahiers de Narratologie}

Analyse et théorie narratives

$23 \mid 2012$

Le sujet et l'art dans la prose française contemporaine (1990-2012)

\title{
L'ekphrasis photographique dans Dora Bruder de Patrick Modiano : entre magnétisme et réfraction
}

\section{Valeria Sperti}

\section{(2) OpenEdition}

\section{Journals}

Édition électronique

URL : http://journals.openedition.org/narratologie/6607

DOI : 10.4000/narratologie.6607

ISSN : 1765-307X

Éditeur

LIRCES

Référence électronique

Valeria Sperti, «L'ekphrasis photographique dans Dora Bruder de Patrick Modiano : entre magnétisme et réfraction", Cahiers de Narratologie [En ligne], 23 | 2012, mis en ligne le 29 décembre 2012, consulté le 30 avril 2019. URL : http://journals.openedition.org/narratologie/6607 ; DOI : 10.4000/ narratologie.6607

Ce document a été généré automatiquement le 30 avril 2019

Article L.111-1 du Code de la propriété intellectuelle. 


\title{
L'ekphrasis photographique dans Dora Bruder de Patrick Modiano : entre magnétisme et réfraction
}

\author{
Valeria Sperti
}

\section{Dora Bruder, un roman d'enquête}

1 Seizième récit de Patrick Modiano, Dora Bruder (1997) reprend le thème fort de son œuvre, celui de l'enquête privée à laquelle la narration confère une dimension historique. Le nom propre, annoncé dans le titre, amorce une recherche existentielle qui implique la protagoniste et qui se réverbère sur l'auteur-narrateur. Le récit retrace, à l'aide de multiples menus indices issus d'un document initiateur - l'annonce de disparition publiée dans Paris-Soir le 31 décembre 1941 - l'histoire d'une juive adolescente et fugueuse qui, après avoir abandonné le pensionnat, s'engouffre dans les brumes obscures du Paris de l'Occupation pour réapparaître à Drancy et connaître le sort de la déportation. Son nom figure dans le premier Mémorial de la déportation des Juifs de France publié par Serge Klarsfeld en $1978^{1}$.

D'emblée, donc, l'écriture modianienne se met à l'écoute de moindres échos de cette histoire et touche ainsi, par les questions de mémoire et d'oubli, aux racines mêmes de la littérature censée offrir, selon l'auteur, un acte de résistance à toute forme d'amnésie, aux effets d'érosion tant du souvenir personnel que de la mémoire collective ${ }^{2}$. L'univers romanesque de l'écrivain prend ici un éclat particulier : circonscrit dans la topographie parisienne et hanté par des sensations minimales, des reconnaissances en fuite, des détails apparemment sans importance, il met au centre l'identité fragilisée d'une jeune inconnue destinée à être broyée par la Grande Histoire, « avec sa grande hache ${ }^{3}$ » (Perec, 1975 : 13). Les rares données biographiques de Dora se superposent, par un effet de surimpression, à celles de son créateur. La fugue, qu'ils partagent, les caractérise symboliquement; le "panier à salade » (Modiano, 1997: 59, 644)-métaphore de la 
connivence empoisonnée entre Allemands et collaborateurs - est aussi l'espace de la rencontre à travers le temps de Dora, de Patrick et d'Albert Modiano - le père mystérieux du narrateur. L'ordre chronologique de l'histoire retraçant la biographie de la jeune cavaleuse s'entrecoupe de temporalités différentes qui renvoient à la longue recomposition de l'enquête de la part du narrateur. L'effet est celui d'une narration intercalée, qui vise à évoquer, par les inquiétudes de l'auteur et ses rapports difficiles avec son père, les mobiles au cœur de l'histoire personnelle de Dora.

3 Les références à la photographie sont un topos du texte et du paratexte modianiens. L'auteur entretient un rapport fort et complexe avec l'image photographique : l'écriture, comparée au « lent travail d'accommodation » (Obaldia) du regard accompli par l'objectif pour une mise au point optimale, doit être aussi légère que la lumière qui s'imprime sur une plaque sensible. Un photographe est le protagoniste d'un de ses romans, Chien de printemps (Modiano, 1993), et ses collaborations d'auteur à des livres de photos, sur Paris notamment, sont connues (Brassaï Modiano: 1999; Modiano: 2003). Pour Patrick Modiano, feuilleter un livre d'images ressemble «à compulser un avis de recherche ", à fixer "l'éphémère pour l'éternité ", à sauver "un visage du néant» (2003: 5). On comprend donc l'affinité de propos entre le déclic photographique et l'écriture littéraire : le cliché de Dora, enfin retrouvé, est « la seule preuve d'un crime qui, sans cela, aurait été effacé par le brouillard et la nuit » (2003:5).

Patrick Modiano, ethnologue des voix urbaines, met en place une écriture légère ${ }^{5}$, à la fois hyperréaliste et onirique, pour retracer les oublis et pour donner une forme à des éléments dérisoires :

Ma démarche n'est pas d'écrire pour essayer de me connaitre moi-même ni de faire de l'introspection. C'est plutôt, avec de pauvres éléments de hasard [...] de trouver un peu de magnétisme à ces éléments qui sont sans intérêt en eux-mêmes, les réfracter à travers une sorte d'imaginaire. (Liban 2003)

Dans Dora Bruder, le magnétisme - les forces attractives reliant les données narratives en un ensemble cohérent - et la réfraction - indiquant les changements de l'écriture qui transforme les diverses données du réel - évoquent deux principes essentiels de la narration. Ils disposent les documents - annonces, extraits, lettres, rapports, photos - en un récit cohérent, en une factographie caractérisée par le souci biographique et l'esprit documentaire ; en même temps, l'écriture rend compte de différents « éléments » qu'elle traverse, leur conférant une cohérence narrative spécifique.

6 Il semble intéressant de suivre la piste ouverte par Modiano et d'observer les effets «magnétiques » et « réfractifs» dans une perspective photographique. Procédant à une étude de la narration de Dora Bruder, telle qu'elle a été publiée dans la première édition, nous analyserons les ekphrasis d'images pour montrer comment l'écriture magnétise et réfracte le cliché, ce document étant, à la différence de la plupart des éléments d'archives cités, absent du texte, décrit sans qu'il y ait reproduction d'image ${ }^{6}$. Nous examinerons ensuite comment la narration relie l'ekphrasis à cet inventaire de documents quelque peu bancal que le narrateur a rassemblé pendant des années et dans lequel l'écriture semble inscrire un possible. Dans Dora Bruder l'image est-elle, suivant les mots de Barthes, « ontologiquement, ce dont on ne peut (rien) dire » (Barthes 2003: 391)? Ou bien est-elle choisie par l'auteur parce qu'elle contient un noyau narratif, un embrayeur de fiction en mesure de faire progresser l'histoire, en la reliant à la diégèse ? Le cliché, simulacre d'une scène réelle, entretient une relation privilégiée avec le temps et l'espace (Méaux 
1997 : 232-3) ; la description de sa contingence devrait permettre au narrateur d'entrer en résonance avec le passé de Dora.

\section{L'acte photographique}

7 Le récit s'ouvre sur le narrateur qui, en 1989, découvre l'annonce de disparition de Dora publiée le 31 décembre 1941. Le XVIIIe arrondissement et le boulevard Ornano - dernière habitation connue de M. et Mme Bruder, une chambre d'hôtel tenant lieu de la précarité des immigrés juifs pendant l'Occupation - sont le théâtre des événements biographiques de l'adolescente et de son narrateur. Les souvenirs personnels de Patrick Modiano s'inscrivent sous le signe photographique par l'évocation d' " un gros photographe au nez grumeleux et aux lunettes rondes » rencontré par l'écrivain quand il se rendait avec sa mère au marché aux Puces de Saint-Ouen. Celui-ci proposait une "photo souvenir» le long de la caserne Clignancourt aux passants qui « ne semblaient pas vouloir se faire photographier» (1997: 10). Un avant-texte de cet épisode se trouve dans l'album photographique de Brassaï publié en 1990, où Patrick Modiano évoque la même rencontre avec le photographe à quelques détails près, « au visage rouge et au costume de plus en plus élimé ", le long de la même caserne, lieu de mémoire important, à préserver car il a hébergé les troupes coloniales et servi de garnison et de terrain d'exercice aux troupes d'occupation : «... il se postait sur l'avenue [...] et guettait les passants qui voulaient bien se faire tirer le portrait " (Brassaï Modiano 1990: 39). L'inversion est significative : le refus de se faire prendre en photo mis en relief dans le roman renvoie à l'impuissance d'authentification, à la dégradation de l'image argentique déjà théorisée et représentée par Roland Barthes (1980) et Hervé Guibert (1981). Selon Jean-Bernard Vray, ce paradigme se relie à la hantise généralisée de la disparition par dilution qui traverse les romans de Modiano favorisant le fantasme de l'absence (Vray 2009: 369). Le texte fait allusion d'ailleurs à une image urbaine manquante : celle du pensionnat de Dora, SaintCœur-de-Marie, disparu pour laisser la place à un complexe résidentiel moderne (Modiano 1997 : 41). Par la suite, l'avant-pénultième séquence du récit relate d'une autre manifestation d'oubli sous le signe de la reproduction visuelle. Sur le haut mur d'enceinte de la caserne des Tourelles - où Dora a été conduite et où les juifs arrêtés étaient interrogés - le narrateur découvre en 1996 une plaque dont il transcrit le contenu: «Zone militaire Défense de filmer ou de photographier» (1997: 132). L'interdiction symbolise la soustraction aux possibilités d'enregistrement, liée au désir collectif d'oubli, à la censure du souvenir. Toutefois, sa retranscription à l'intérieur de Dora Bruder témoigne que l'amnésie ne peut pas être totale et que la mauvaise conscience du présent, qui veut que tout ce passé soit oublié, cède à l'écriture qui en pérennise le souvenir dans sa précarité (Ceserani 2011: 221). Les trois éléments narratifs de l'impossibilité photographique - le refus personnel des passants sur le trottoir de l'avenue de Clignancourt, l'image manquante du pensionnat de la rue de Picpus et l'interdiction officielle affichée sur le mur de la caserne des Tourelles - montrent l'impuissance du document photographique devant la reconstruction de l'histoire de Dora. Mais leur cohérence narrative réside dans l'importance que le narrateur leur confère comme jalons d'une métaenquête qui prend le pas sur les résultats de l'enquête elle-même parce qu'elle en sanctionne les difficultés et les limites, et qu'elle offre au narrateur des occasions pour associer ses souvenirs et ses pensées aux souvenirs et aux pensées manquants de Dora. 


\section{Portraits et photos de famille : pouvoir du magnétisme et de la réfraction}

8 La vie de Dora Bruder est inconnue et le narrateur la reconstruit à travers les documents qu'il retrace pendant huit années, en laissant apparaître les lacunes. Parfois il remplit les vides de l'existence de l'adolescente par la narration d'épisodes autobiographiques et de situations similaires vécues par son père dans le Paris occupé. Parfois il insère des documents littéraires, des lettres testimoniales, des références intertextuelles. La narration est ainsi réfractée sur des plans temporels divers. L'impression est que, par Dora, le narrateur procède à se «créer un passé et une mémoire avec le passé et la mémoire des autres »(Berl Modiano 1976: 9). Dans la diégèse, donc, les ruptures temporelles de l'ordre chronologique facilitent les effets d'osmose entre Dora et le narrateur?

9 Les photos font partie du dossier documentaire mis en place dans le roman. À la différence de documents écrits, qui sont pour la plupart retranscrits à l'intérieur du récit, les images ne sont pas reproduites, mais racontées. Les photos de Dora et de sa famille sont regroupées au moment où Patrick Modiano, après avoir découvert l'acte de naissance de Dora et l'acte de mariage de ses parents, manque d'autres informations importantes sur le travail d'Ernest et Cécile, l'école maternelle de Dora, sur leur vie d'immigrés juifs avant l'Occupation. Alors que tout bascule, il a une certitude topographique, la porte de Clignancourt. Contraint d'avouer un trou temporel et informationnel, un blanc dans l'écriture que la fiche d'Ernest dressée pendant l'Occupation et le témoignage direct de sa nièce ne dissipent pas - «Et les années se sont écoulées [...] Je ne sais rien d'eux, au cours de ces années » (1997: 31) -, le narrateur procède à l'ekphrasis de huit images, comprenant des photos de famille et des portraits. Leur description occupe un chapitre à part $^{8}$; elles se juxtaposent sans solution de continuité typographique suivant l'ordre chronologique, de la plus ancienne à la plus récente, excepté la dernière image, qui propose une analepse. Celles-ci appartiennent au passé de Dora, mais elles sont décrites à partir du moment de l'énonciation, conférant au passé une fluidité sans points d'appui, marquée par l'indicatif présent, les indéfinis («quelques photos [...] une photo [...] une autre photo») et les déictiques (Schulte Nordholt 2012: 531). Le ton dubitatif est renforcé par la présence d'éléments modalisateurs - points d'interrogations, conditionnels, adverbes d'opinion- qui atténuent toute certitude quant à l'amplification du texte de la dimension informative à l'explicative.

10 Aux phrases nominales qui ouvrent la séquence ekphrastique («Quelque photos de cette époque. La plus ancienne, le jour de leur mariage » 1997 : 32) s'ajoute sur le plan de la signification le manque de renseignements sur la provenance des images. Ces éléments contribuent à accentuer le caractère indéfini, imprécis des clichés ; l'absence de verbe les relie, par leur sécheresse informative, aux fiches administratives et les caractérise comme des épaves de l'oubli. Le premier instantané est celui du mariage des parents de Dora scandé en trois syntagmes, purement dénotatifs: ils renseignent sur la pose photographique du couple, le voile de l'une et l'habit de l'autre (1997: 32-3). Suit, sans solution de continuité, un autre cliché, tout aussi conventionnel et chronologiquement conséquent: le même couple avec Dora au centre. L'ekphrasis est complétée par une indication concernant l'enfant : « elle n'a pas plus de deux ans » dont l'imprécision laisse 
flotter l'indétermination. Comme pour les images suivantes, l'ekphrasis se focalise sur la pose, déterminant pour chaque cliché le membre de la famille photographié ainsi que sa position face à l'objectif. Il en est ainsi pour la troisième et la quatrième photo, prises à l'occasion d'une remise de prix, où Dora figure, un livre à la main, avec une robe et des socquettes blanches debout près de sa mère, en train de poser sa main droite sur son épaule, geste qui symbolise leur lien de consanguinité. La contingence de ces images, célébrant les étapes importantes de la vie du jeune couple juif émigré à Paris, puis de leur fille, est diluée dans le stéréotype: dans leur banalité, les moments clou d'une vie familiale n'enlèvent rien à l'oubli du passé qui a englouti l'adolescente. Ces ekphrasis sont le témoignage d'une "normalité » qui sera bientôt effacée par les vicissitudes personnelles et historiques dans lesquelles la jeune fille en cavale se trouvera impliquée. Toutefois aucune notation n'insiste sur les visages qui restent opaques, sans forme propre, pourvus seulement de signes identitaires : Dora, la mère, le père... La description évite de s'épancher sur leur aspect physique. Quand, dans le cinquième instantané - « une autre photo de Dora et de sa mère » $(1997: 33)$ - sont mentionnés les cheveux plus courts de la jeune fille, le lecteur n'a pas de points de repères comparatifs, et la référence à la photo précédente reste sans appui visuel. Le rituel photographique est souligné par l'insistance du narrateur sur la pose et le décor; l'ekphrasis de la cinquième photo s'étend sur la présence d'un fond, « qui doit être le panneau du photographe » et dans les images de la distribution des prix, sur un « cube blanc [qui] doit être là pour le décor » (1997 : 33). Patrick Modiano accentue l'artifice du cliché révélant le caractère conventionnel de la photo-souvenir, de l'image qui n'interpelle que les membres de la famille, les amis, enfin tous ceux qui ont disparu. Les ekphrasis se connotent par une réduction sur le plan descriptif et une atténuation sur celui des émotions. Toute trace du choc du narrateur face à la vérité de l'image, toute réflexion sur la présence « en chair et en os » dans les clichés, tout désarroi face à leur « ça-a-été » sont absents (Barthes 1980 : 124).

11 Si la séquence scande, par sa cohérence narrative, la représentation des étapes successives de la vie familiale des Bruder, le narrateur évite toute personnalisation dans l' ekphrasis. Cette dernière est précédée d'une citation proleptique extraite de la fiche d'Ernest Bruder, assumée par le narrateur à la première personne : «Sur la fiche de lui qui a été faite pendant l'Occupation et où j'ai lu...» (Modiano 1997: 32). Dans la description des images, par contre, le ton impersonnel est éclatant : " on reconnait, on dirait, on ne distingue pas... ». Il n'y a aucune référence au regard du narrateur, au «j'ai vu » que l'on s'attendrait à retrouver ici.

12 Dans la présentation des témoignages, les photos sont incluses dans le texte sous forme d' ekphrasis et regroupées, magnétisées par des articulations introductives et des déictiques pronominaux («Quelques photos, la plus ancienne... ») comme des preuves de l'enquête. La présence de dubitatifs déjà évoquée accentue l'atmosphère d'imprécision : « environ, ce qui semble, peut-être, [il] doit être là... ». Si la séquence est cohérente sur le plan de la signification et sur celui de l'énoncé par l'évocation sub specie photographique de la disparue - les huit photos énumérées une par une - et par la répétition anaphorique du sujet, la description en ressort décousue. Au style paratactique, elliptique, riche en phrases nominales, correspond une description impersonnelle qui se fixe sur des détails extérieurs, sur les vêtements, sur les poses, jamais sur les visages.

13 L'ekphrasis joue sur l'effet de liste et sur le vertige du bloc typographique compact qui contient l'ekphrasis de six premiers clichés : « Une photo... Une photo de Dora... Une autre photo... Une autre photo de Dora et de sa mère... Une photo de forme ovale... une 
photo...» (1997 : 32-4). Cette énumération n'apprend rien au lecteur sur les visages; les expressions restent opaques, suspendues dans un présent intemporel, un non-temps qui indique les éléments, les enveloppant d'une atmosphère détachée sur le plan émotionnel. Toutefois, dans les deux dernières descriptions, qui renvoient à des clichés pris en plein air, donc plus spontanés, le lecteur peut reconnaître, bien qu'estompé, le punctum barthésien, c'est-à-dire la trace du détail irrationnel qui laisse percevoir l'implication affective du spectator. Le narrateur évoque, dans le cliché qui représente Cécile, une présence : « au fond, la silhouette d'un enfant, de dos, les jambes et les bras nus, en tricot noir ou en maillot de bain. Dora?» (1997: 34). Dans l'ekphrasis la silhouette fonctionnerait de punctum atténué : dans son imprécision, elle apparaît déjà chargée de son destin de spectre et l'incertitude du narrateur le met en relief.

Le dernier cliché de cette séquence tranche sur la liste précédente par le temps évoqué «Une photo plus ancienne de Dora seule, à neuf ou dix ans» (1997: 34) - qui est antérieur, et parce que sa description occupe un paragraphe à part. L'enfant est illuminée par un rayon de soleil, le pied droit appuyé sur un rebord de béton de ce que le narrateur suppose être une volière. Le détail saisissant se cache derrière la difficulté à distinguer les formes en raison de l'effet de clair-obscur du cliché : « ... elle a posé le pied droit sur le rebord de béton de ce qui pourrait être une grande cage ou une grande volière, mais on ne distingue pas, à cause de l'ombre, les animaux ou les oiseaux qui y sont enfermés. Ces ombres et ces taches de soleil sont celles d'un jour d'été » (1997: 34). L'insistance de la description sur le clair-obscur est le négatif ekphrastique de la difficulté du narrateur de comprendre le secret de l'existence de Dora.

Le regard du narrateur ne s'arrête jamais sur les visages, ne fait aucune allusion aux sourires, aux expressions, aux yeux; il enregistre impersonnellement les indices dénotatifs, les stéréotypes sociaux de l'époque. Cette suite d'ekphrasis, d'apparitions fugaces représente la famille Bruder comme trois spectres, figures sans avenir; leur inscription dans la durée est atténuée par le manque d'un avant et d'un après qui se heurte aux blancs des informations oubliées. Dépourvues d'éléments connotatifs, ces descriptions plates et opaques où dominent le noir et le blanc transmettent au lecteur la difficulté de leur attribuer une cohérence interprétative. Pourquoi alors le narrateur bloque-t-il toute effervescence imaginaire, pourquoi aplatit-il toute sémiologie photographique ${ }^{9}$ dans ces ekphrasis? L'absence de réfraction fictionnelle dans la description des images est d'autant plus surprenante si nous nous rappelons que ce sont des détails - les rues du XVIIIe arrondissement, une voiture cellulaire - qui amplifient la diégèse introduisant de prolepses autobiographiques ou d'épisodes fictifs dans le récit. Les huit ekphrasis se configurent ainsi comme un trou noir, opaque, que l'écriture traverse. Elles observent, constatent sans réfracter par le commentaire ou l'imaginaire : la seule description physique de Dora doit rester celle de l'avis de recherche liminaire. Ainsi ces images stéréotypées d'avant le drame - car, à s'en tenir à la chronologie du narrateur, elles datent de 1938 au maximum - sont-elles «indialectiques», selon la définition de Roland Barthes ${ }^{10}$. Leur ekphrasis, appauvries d'éléments connotatifs et plongées dans un présent monstrueux, figé et inauthentique, restitue par l'écriture la valence de la photo comme " le théâtre mort de la mort, la forclusion du Tragique; elle exclut toute purification, toute catharsis» (Barthes 1980:141). La cohérence narrative rassemble comme des aimants ces descriptions de clichés, introduites pour combler un vide documentaire; leur imperméabilité à la transformation de leurs indices par l'interprétation du narrateur les immobilise dans le temps. La platitude réductive de leur 
ekphrasis, où l'alternance de noir et de blanc est un renvoi tautologique à la photo, accompagnée de la réticence que l'on cueille dans les rares et discrètes allusions au détail émotif contrastent avec le bouleversement que l'auteur a exprimé à plusieurs reprises dans sa correspondance avec Klarsfeld à la vue des photos de Dora et des parents (Modiano-Klarsfeld 2012). Le trou émotif concourt à généraliser le drame des Bruder, à en élargir la portée d'une famille juive de l'Europe de l'Est immigrée à Paris à tout un peuple ${ }^{11}$. Mais cette discontinuité entre la cohérence textuelle des images et leur résonance à vide dans la diégèse nous semble importante.

Une seule image de Dora sera encore décrite (1997 : 92-4), échappant au magnétisme des photos d'avant l'Occupation, parce que le narrateur l'a récupérée successivement et qu'elle est différente. Toutefois, comme les précédentes, elle vient remplir un vide dans l'enquête du narrateur sur la vie de Dora: «Il n'y a aucune trace d'elle entre le 14 décembre 1941, jour de sa fugue, et le 17 avril 1942 où, selon la main courante, elle réintègre le domicile maternel, c'est-à-dire la chambre d'hôtel du 41 boulevard Ornano " (1997: 90). Et encore, comme pour les précédentes, sa provenance reste vague et mystérieuse ( J'ai pu obtenir il y a quelques mois une photo...») et les informations pourvues relèvent du tâtonnement, de l'hypothèse :

Sans doute la dernière qui a été prise d'elle [...] Je ne sais pas à quelle date a été prise cette photo. Certainement en 1941, l'année où Dora était pensionnaire au Saint-Cœur-de-Marie, ou bien au début du printemps 1942 quand elle est revenue, après sa fugue de décembre, boulevard Ornano (1997 : 92-3).

Bien qu'il s'agisse encore d'un portrait familial s'étendant sur trois générations - la grand-mère, Cécile et Dora côte à côte - on y remarque une différence d'atmosphère. Alors que les premières images étaient innocentes, cette dernière est hantée par les inquiétudes de l'adolescente et par le climat sombre de l'Occupation (Schulte-Nordholt 2012 : 535-7). L'ekphrasis détaille longuement les éléments descriptifs, qui cette fois-ci sont intercalés par la représentation d'indices significatifs : à partir des expressions du visage de Cécile et de la grand-mère - «Les deux femmes ne sourient pas » (1997: 93) pour se focaliser ensuite sur Dora et commenter certains signes sur sa figure : « Elle tient la tête haute, ses yeux sont graves, mais il flotte sur ses lèvres l'amorce d'un sourire. Et cela donne à son visage une expression de douceur triste et de défi » (1997: 93). Le narrateur souligne dans cette ekphrasis l'irruption de la contingence, qui contient la réalité et le passé interprété à la lumière du futur et qui pointe à la condition mortelle des trois femmes. La description se clôt par une série de questions, quatre points interrogatifs, des appels lancés dans le vide, qui s'attachent à autant de détails oubliés, désormais sans importance : «Qui a bien pu prendre cette photo ? Ernest Bruder? Et s'il ne figure pas dans cette photo, cela veut dire qu'il a déjà été arrêté ? [...] Dora porte-t-elle la jupe bleu marine indiquée sur l'avis de recherche? (1997 : 93). Cette ekphrasis partage avec la séquence de huit clichés la présentification de la trace à l'intérieur d'une écriture plate, transparente qui renforce l'opacité du monde de Dora: «Elle est en compagnie... elle porte des bas... elle tient la tête haute... ». Cette présentification est importante au niveau de la narration parce qu'elle intensifie un effet de simultanéité : la photo semble décrite au moment de l'énonciation et de ce fait les questions la rattachent à la métaenquête, au présent de la remémoration. Ce temps devrait accentuer la participation directe, émotive de l'énonciateur. La réflexion qui en découle insiste toutefois sur la perspective temporelle : « Les temps de la photo, ils étaient protégés quelques secondes et ces secondes sont devenus une éternité » (1997 : 93). L'usage du déictique personnel « ils » délie la référence aux trois femmes décrites dans l'ekphrasis; il contribue à élargir le 
sentiment de la perte non seulement au destin de Dora et de sa famille, mais à toutes les victimes de l'Occupation.

Deux occurrences ekphrastiques dans Dora Bruder méritent encore une mention, même si elles ne concernent ni l'adolescente en cavale, ni sa famille. Dans la diégèse elles sont postérieures aux portraits familiaux et à la représentation inquiète de la jeune Dora. C'est le printemps 1942 et le chapitre retranscrit plusieurs documents dont un, le premier, concernant Dora. Il prouve que l'adolescente, après des fugues successives, avait été remise à sa mère le 15 juin 1942. Dans la même fiche, il est conseillé de demander l'aide des "assistances sociales de la police (quai de Gesvres) [qui] feraient le nécessaire " (1997: 103), c'est-à-dire la faire admettre dans une maison de redressement pour l'enfance. Mais le manque d'informations supplémentaires s'inscrit dans le texte par des questions et des points d'interrogation successifs et la narration détaille, en retranscrivant l'ordonnance, les restrictions de plus en plus sévères imposées aux Juifs, notamment le port de l'étoile jaune, suivie des fiches des transgresseurs, sans doute oubliées par mégarde dans les archives. Le narrateur assume ici dans sa fonction d'enquêteur celle de sentinelle du souvenir, citant des rapports complets de jeunes qui, pris dans les filets de la police française, "passèrent par le Dépôt et Drancy, avant Auschwitz» (1997: 106). Pour rendre la connivence vénéneuse des Années Noires, il décrit une photo de l'époque représentant deux assistantes sociales de la police. L' ekphrasis est indicielle, désignant l'âge et l'uniforme des femmes. Mais le narrateur souligne que même le cliché testimonial manque à sa fonction informative : la tête et le calot d'une assistante cachent les inscriptions derrière elles et notamment le numéro du bureau des inspecteurs. Le commentaire laconique - «On ne saura jamais le numéro de cette porte » $(1997: 110)$ - révèle un paradoxe : ce numéro est illisible, mais il ne servirait qu'à accentuer l'inanité des informations transmises par l'image (Xanthos $2011: 218$ ). Ici toutefois l'ekphrasis, dont la portée narrative et fictionnelle est nulle, a une fonction référentielle et métonymique évidente. Avec les rapports de police, l'ordonnance et les fiches elle donne corps à la tragédie collective de l'Histoire dont a été victime Dora. Par le biais de ce petit détail apparemment sans importance, le narrateur montre la collusion toujours à l'affût entre l'objet photographié et l'oubli.

Dans la dernière occurrence le narrateur, relatant un épisode autobiographique qui remonte à ses vingt ans, passe rapidement en revue trois images du IVe arrondissement entre l'Église Saint-Gervais, la rue des Jardins-Saint-Paul, la rue Nonnains-d'Hyères et la Seine (Modiano 1997 : 138). Leur cohérence narrative réside dans la séquence sérielle de l' ekphrasis qui passe des immeubles encore existants, à ceux à moitié détruits jusqu'à un terrain vague. Démolis à la suite d'un réaménagement urbain qui n'est pas directement lié aux Années Noires, les immeubles symbolisent l'effacement de l'oubli, et leur ekphrasis la conservation de l'image, là où il n'existe plus de trace ; conservation dont la description, limitée à l'indication de l'emplacement, amplifie le sentiment de perte, de dépaysement :

De nouveau je ressentais un vide. Et je comprenais pourquoi [...] Les lambeaux de papiers peints que j'avais vus encore il y a trente ans rue des Jardins-Saint-Paul, c'étaient les traces de chambres où l'on avait habité jadis - les chambres où vivaient ceux et celles de l'âge de Dora que les policiers étaient venus chercher un jour de juillet 1942. La liste de leurs noms s'accompagne toujours des mêmes noms de rues. Et les numéros des immeubles et les noms des rues ne correspondent plus à rien (1997 : 138-9). 


\section{Ekphrasis et transcription documentaire}

Lorsque, à l'automne 1940, la narration de la vie de Dora se perd dans les méandres noirs du pensionnat, Patrick Modiano écrit que si elle y était restée, elle aurait pu échapper à son destin. Il revient ensuite au moment de l'énonciation, à l'automne 1996, et mentionne la lecture du cinquième et sixième livres des Misérables. C'est le récit de la traversée nocturne de Paris que font Cosette et Jean Valjean, poursuivis par Javert, où Victor Hugo nomme le 62 de l'avenue de Picpus, la même adresse que le pensionnat de Dora Bruder. Une longue citation du roman hugolien comble par la référence intertextuelle l'absence informative sur le séjour de Dora et l'absence visuelle, puisque le bâtiment a été démoli entre temps: "Nous n'avons pu passer devant cette maison extraordinaire, inconnue, obscure, sans y entrer et sans y faire entrer les esprits qui nous accompagnent et qui nous écoutent raconter, pour l'utilité de quelques-uns peut-être, l'histoire mélancolique de Jean Valjean » (1997: 53). C'est un de nombreux intertextes de substitution, que Modiano enchâsse dans le récit. D'autres renvois à Voyage de noces (Modiano 1990), roman écrit par l'auteur en mal de traces de Dora, ont été inconsciemment inspirés par la même topographie urbaine, les petites rues autour du pensionnat. Patrick Modiano en train de se pencher sur le destin de Dora, de rassembler les traces éparses est un thème du récit aussi important que la biographie de Dora. Le récit est avant tout l'histoire d'une enquête en cours, qui avance à coups d'ekphrasis, de citations de documents officiels et littéraires. En ce sens, les changements apportés par l'auteur dans l'édition Folio, deux ans après la publication de la première édition de 1997, ainsi que la publication de quelques images dans les éditions traduites, prouvent que l'appréhension biographique de Dora est un processus encore in fieri (Morris, 2000 : 273). Tout ce matériel hétérogène, sans cesse précisé, concourt à étoffer, à couvrir le laps de temps, huit mois, séparant l'avis de recherche de l'internement de l'adolescente au camp de Drancy le 13 août 1942, tout autant qu'à représenter les huit ans passés entre la découverte de Dora et son énonciation dans le récit. Dans son appréhension biographique de Dora par l'écriture, trouver des traces, récupérer des documents, est pour Patrick Modiano une activité indissolublement liée à celle de laisser résonner les vides. Les références littéraires intertextuelles concourent à ce procédé de réverbération et accordent les thèmes forts du roman: l'enquête, la métaenquête et la biographie de Dora ${ }^{12}$. Une autre référence intermédiale est la mention du film Premier rendez-vous, tourné au début de l'Occupation. Son intérêt n'est pas dans le fait que le film est une version « douce et anodine» de la fugue d'une jeune fille, mais dans l'écriture en résonance de Modiano qui capte et décrit dans la pellicule les regards des spectateurs de l'Occupation et de Dora sans doute : «Et tous ces regards, par une sorte de processus chimique, avaient modifié la substance même de la pellicule, la lumière, la voix des comédiens. Voilà ce que j'avais ressenti, en pensant à Dora Bruder, devant les images en apparence futiles de Premier rendez-vous " (1997: 82). Il semble important de souligner que le narrateur utilise des témoignages visuels d'où tout pathos est absent pour atteindre la compréhension de Dora. Pour que cette compréhension ait lieu, il doit narrativiser l'absence de connotation émotionnelle des photos, le gommage de l'atmosphère de l'Occupation dans le film. Ce n'est que dans ce creux, dans cette réfraction à vide, que Modiano peut se mettre à l'écoute de Dora et en restituer le souvenir. 
21 Ainsi, oscillant entre une énonciation qui magnétise les éléments documentaires épars et qui les réfracte en laissant apparaître toute l'incohérence, les blancs et les vides, le fantasme du souvenir s'amplifie-t-il à l'évocation d'autres personnes arrêtées à la même période de Dora, dont les noms et les fiches composent une litanie funèbre posthume et à celle de trois écrivains : «Beaucoup d'amis que je n'ai pas connus ont disparu en 1945, l'année de ma naissance » (1997: 100). L'allemand Friedo Lampe, dont le premier roman Au bord de la nuit avait été séquestré et qui est mort fusillé par erreur par les soldats de l'Armée Rouge en 1945. L'extrait d'une de ces lettres révèle une sensibilité proche de celle de Modiano dans l'ambition de «rendre sensibles quelques heures, le soir, entre huit heures et minuit, aux abords d'un port» (1997: 95). Ensuite Felix Hartlaub, historien allemand cultivé, responsable des archives françaises pendant l'Occupation : de son livre, le narrateur cite sa description d'un bordel de la rue de Clichy où « tout baigne dans une lumière étrange d'aquarium tropical, de verre surchauffé » (1997 : 97). Ils appartiennent à l'autre côté de la mémoire collective. Quant à Roger Gilbert-Lecomte, un des animateurs du Grand Jeu, ses vicissitudes sont évoquées parce qu'elles s'enchevêtrent avec celles de Dora, une amie du poète ayant été déportée une semaine avant elle (1997: 99). Ces épisodes de substitution témoignent d'une opacité autour de Dora que rien ne peut éclaircir, et affichent aussi un refus de la transparence idéologique du récit victimaire et la volonté de représenter toute l'épaisseur de l'Occupation, avec ses connivences et ses formes collaborationnistes. La lettre que Patrick Modiano déclare avoir trouvée par hasard dans une librairie des quais mérite une mention à part. Dans celle-ci, un inconnu, du nom de Robert Tartakovsky interné à Drancy, s'adresse à sa famille, à la veille de son départ pour les camps d'extermination (1997: 123-9). Sa reproduction dans le récit réfracte et universalise la condition de Dora, sur laquelle le narrateur n'a aucun renseignement. Elle devient ainsi une mise en abyme, par lettre interposée du fatum de l'adolescente.

Construire un récit sur des documents authentiques, mais fragmentaires, cultiver une narration intercalée d'intertextes va de pair chez Modiano avec la hantise du blanc; le remplissage littéraire où la cohésion narrative produit et conjure le vide, s'abandonne à l'oubli tout en le contrastant (Douzou 2010 : 310). La banalisation accordée à la mise en texte des photos est sans doute liée au thème de la disparition. Sa description en pérennise la trace dans la présentification. Les photos contribuent à la "momification » de Dora et la prédestinent à l'exhumation à venir, sous forme d'ekphrasis et de récit littéraire. Cette opération contradictoire correspond aux deux conceptions de l'énigme à l'œuvre chez Modiano : énigme de l'incomplétude et celle du suivi de la trace, qu'il s'agit « d'ourdir et de dire » plus que de résoudre (Meyer-Bolzinger $2007: 239$ ).

\section{Conclusion}

La textualisation de la trace argentique en tant qu'image testimoniale n'a ni de vertu romanesque, ni le pouvoir de reconfigurer le temps. Elle renvoie à une narration à la fois en construction et consciente de se heurter à son impossibilité. Les ekphrasis du portrait de Dora décontextualisent le visage, l'enlèvent à la référence spatio-temporelle, le figeant dans un présent absurde, monstrueux, qui rappelle la taxonomie des Mémoriaux de Klarsfeld, son archétype (Douzou 2010 : 302).

Les documents et les intertextes vicaires sont une citation du passé, alors que les images, dans le passage du registre visuel à la prose, sont actualisées dans une forme narrative qui 
est réductive, aplatie et qui volontairement se détourne des indices susceptibles d'être amplifiés par l'écriture. La présentification de l'ekphrasis unie à l'impersonnalisation du regard semblerait empêcher toute transformation littéraire. Le lecteur insère l'image dans le statut documentaire mis en œuvre par le narrateur, mais il ne peut ni relever ni partager les éléments connotatifs. L'ekphrasis devient ici métonymique du passé absent auquel le narrateur ne peut plus avoir accès; par son aphasie émotive elle réfracte l'opacité de la réalité qu'elle serait censée représenter et met en relief les conséquences du procès d'oubli. Dans sa platitude elle renvoie à un manque et en même temps à l'impossibilité ontologique de remplir ce manque. En ce sens Patrick Modiano met en œuvre un renversement des expédients du roman policier pour confondre les pistes et révéler l'inanité de tout témoignage.

Dora Bruder, suivant le mince fil biographique de cette adolescente, fait allusion aux ambiguïtés et aux compromissions de l'époque. Toutefois il n'y a pas de déterminisme idéologique : le récit dit l'ébahissement face à une histoire composée de photos muettes, privées d'indices, de fichiers où ce qui importe reste dans le non-dit. En ce sens la textualisation photographique, tout en affichant une cohérence narrative au niveau de l'énonciation, contribue à montrer une densité différente par rapport aux autres témoignages et à dévoiler le rôle privilégié que Patrick Modiano lui accorde : par sa raréfaction connotative elle apporte sa contribution à la configuration de l'environ, du sans doute, de l'à peine aperçu. L'énonciation ekphrastique se compose d'ellipses et d'anadiploses; elle ne prolonge pas ce que l'instant a fixé, au contraire, elle réfracte les différences, laissant entrevoir les fissures; elle souligne les séparations dans leur juxtaposition cohérente dans la narration. Ces textualisations sont comme des épaves dans l'oubli, des images en prose qui surnagent sur le présent de la narration. L'Occupation devient un lieu mental, une position à partir de laquelle Modiano sonde et évoque les événements: l'image argentique est une composante essentielle de cette position. Malgré la réticence interprétative des signes et des images, qui pour cela s'inscrivent sous le signe de la déception, le narrateur a ouvert et réparé par le récit lacunaire de cette inconnue une blessure collective et individuelle. Ces ekphrasis sont de la pure dénotation, elles contribuent à faire de l'Occupation un fatum, une menace, un lieu de mémoire (Sperti, 2005 : 165). La diégèse réduit la force d'impact des clichés et laisse la parole aux documents, aux registres, aux mains courantes, sans axiologie, ni implication affective. La platitude descriptive des portraits familiaux réside dans l'absence de regard individuel, de détails qui singularisent le hic et nunc de Dora et dans cette présentification de l'image qui estompe le "ça-a-été». Mais la force de cette platitude, voulue par l'auteur, dont l'ekphrasis offre un exemple saisissant, est aussi celle de l'écriture qui laisse résonner le trou documentaire et le vide des images qui s'obstine à écrire et à rendre les difficultés d'écrire ces absences un thème privilégié du récit. Par cette réflexivité métafictionnelle Patrick Modiano s'interroge sur le statut fictionnel du récit, sur ses rapports avec la réalité documentaire. Par l'image d'archives et la photo de famille il donne naissance à son personnage Dora et pérennise avec elle le destin de ceux que les «sentinelles de l'oubli» (Modiano 1997: 18) avaient condamnés à disparaitre de la mémoire collective. stylistique aux différentes typologies de témoignage - intermédial, fictif et administratif tout indique le récit en train de se construire, les repères parsemés sur la table de travail 
en attendant la pièce manquante, tout ce qui, magnétisé par la narration, transforme ce récit réfracté dans l'appréhension fraternelle, visionnaire et lacunaire d'une inconnue.

\section{BIBLIOGRAPHIE}

BAL, Mieke, 2003, « Atti di sguardo : Proust, il romanzo e la memoria visiva», dans Franco Moretti (dir.), Il romanzo. Temi, luoghi, eroi, Torino, Einaudi, p. 279-291.

BARTHES, Roland, 1980, La chambre claire. Note sur la photographie, Paris, Gallimard, Cahiers du cinéma.

BARTHES, Roland, 2003, " Proust et la photographie», in La préparation du roman I et II. Cours et séminaires au Collège de France (1978-1979 et 1979-1980), Paris, Seuil-Imec, p. 389-457.

BERL, Emmanuel, MODIANO Patrick, 1976, Interrogatoire, Paris, Gallimard.

BLANCKEMANN, Bruno, 2009, Lire Patrick Modiano, Paris, Armand Colin.

BRASSAI, MODIANO, Patrick, 1990, Paris tendresse, Paris, Hoëbeke.

CESERANI, Remo, 2011, L'occhio della Medusa. Fotografia e letteratura, Torino, Bollati Boringhieri.

CIMA, Denise, 2003, Étude sur Patrick Modiano : Dora Bruder, Paris, Ellipses, « Résonances ».

DOUZOU, Catherine, 2010, « Du blanc de la mémoire aux blancs du texte», dans Modiano ou les intermittences de la mémoire, sous la dir. de Anne-Yvonne Julien, Paris, Hermann, p. 302-312.

GUIBERT, Hervé, 1981, L'Image fantôme, Paris, Minuit.

KLARSFELD, Serge, 1978, Le Mémorial de la déportation des Juifs de France, Paris, Klarsfeld.

KLARSFELD, Serge, 2001, La Shoah en France 4. Le Mémorial des enfants juifs déportés de France, Paris, L'Association « Les Fils et Filles des déportés juifs de France » and the Beate Klarsfeld Foundation.

LIBAN, Laurence, 2003, « Modiano. Entretien», dans Lire, octobre http://www.lexpress.fr/ culture/livre/modiano_808386.html

MEAUX, Danièle, 1997, « La photographie et le temps. Le déroulement temporel» dans L'image photographique, Aix en provence, Publications de l'Université de Provence.

MEYER-BOLZINGER, Dominique, 2007, « Investigation et remémoration : l'inabouti de l'enquête chez Patrick Modiano» dans Christelle Reggiani, Bernard Magné (dir.), Écrire l'énigme, Paris, Presses Universitaires de Paris Sorbonne, p. 231-239.

MODIANO, Patrick, 1990, Voyage de noces, Paris, Gallimard.

MODIANO, Patrick, 1999, Dora Bruder, Paris, Gallimard, « Folio ».

MODIANO, Patrick, 1997, Dora Bruder, Paris, Gallimard.

MODIANO, Patrick, 1999b, « Les objets du siècle, Libération, 13 mars.

MODIANO, Patrick, 2003, « Préface» dans CHARDIN, Virginie, Paris et la photographie, cent histoires extraordinaires de 1839 à nos jours, Paris, Éditions Parisgramme. 
MODIANO Patrick, KLARSFELD, Serge, 2012, « Correspondance» dans Patrick Modiano Cahiers de l'Herne, dir. Maryline Heck et Raphaëlle Guidée, Paris, Editions de l'Herne, p. 178-186.

MORRIS, Alan, 2006, « Avec Klarsfeld, contre l'oubli : Patrick Modiano's Dora Bruder », Journal of European Studies, $\mathrm{N}^{\circ} 36$, p. 269-293.

OBALDIA, Bernard (dir.), Dictionnaire Patrick Modiano, entrée "Rafistoler,/Bricoler", http://littand-co.org/au_temps/autemps_r.htm

PEREC, Georges, 1975, W ou le souvenir d'enfance, Paris, Denoël.

SCHULTE NORDHOLT, Annelies, 2012, « Photographie et image en prose dans Dora Bruder de Patrick Modiano », Neophilologus, n 96, vol. 4, p. 523-540.

SPERTI, Valeria, 2005, Fotografia e romanzo. Marguerite Duras, Georges Perec, Patrick Modiano, Napoli, Liguori.

VRAY Jean Bernard (2009), « Noirceur de Modiano. Disparition et Photographie», in Roger-Yves Roche (dir.), Lectures de Modiano, Nantes, Editions Cécile Defaut, p. 347-370.

XANTHOS Nicolas (2011), « Sentinelles de l'oubli et reflets furtifs, Permanence, rupture et régimes d'historicité dans Dora Bruder », Orbis Litterarum, n 66, vol. 3, p. 215-237.

\section{ANNEXES}

Valeria Sperti est professeur de littérature française au Dipartimento di Scienze Umane de l'Université de la Basilicate en Italie. Ses intérêts de recherche portent sur le statut de la reproduction photographique dans le roman français moderne et contemporain, sur les enjeux de la mémoire et de l'autobiographie dans la littérature française contemporaine et sur le choix autotraductif des écrivains expatriés. Elle a publié trois volumes (Ecriture et mémoire, 1999 ; La parola esautorata, 2000 ; Fotografia e romanzo, 2005) et des études sur Marguerite Duras, Nancy Huston, Henri Lopes, Patrick Modiano, Georges Perec, Sony Labou Tansi et Marguerite Yourcenar entre autres.

\section{NOTES}

1. Serge Klarsfeld a envoyé à Patrick Modiano plusieurs renseignements sur Dora Bruder et les portraits de famille qu'il décrit.

2. Pour une vision d'ensemble des thèmes et des enjeux de l'œuvre, voir Cima (2003) et Blanckemann (2009).

3. De nombreuses références intertextuelles implicites relient Dora Bruder à $W$ ou le souvenir d'enfance de G. Perec (1975). Voir Morris 2006 : 277.

4. Arrestation du père de Modiano en 1942 et arrestation du père et du fils par association, années 60 .

5. «Il peut lui aussi [le stylo] peser des tonnes, et toute une vie est parfois nécessaire pour essayer de rendre son stylo aussi léger que le rêve d'une caméra légère » (Modiano, 1999b).

6. La première édition, celle de la Collection Blanche de 1997, se présente sans images. L'édition Folio (1999) porte une image de René-Jacques en couverture; la traduction américaine apporte des précisions textuelles et deux reproductions d'images face aux pages où elles sont décrites. L'édition japonaise ajoute aux images la reproduction de plusieurs documents. Voir Alan Morris (2006 : 270-2) et Annelies Schulte Nordholt (2012 : 527) qui remarque que l'ouverture graduelle 
aux photos répond à un besoin d'images et va de pair avec une graduelle transformation du récit de roman en document biographique.

7. Fraternité $=$ Bruder $=$ Rudy

8. La scansion des chapitres et des paragraphes fait référence à la première édition. Des variations apparaissent dans l'édition Folio (1999).

9. Sur la platitude de l'image, voir Mieke Bal (2003).

10. Sur cette question je me permets de renvoyer à mon ouvrage (Sperti $2005: 129-66)$

11. Voir l'analyse pertinente de Schulte Nordholt $(2012: 538)$ qui attribue un regard posthume aux ekphrasis de Dora Bruder.

12. Sur la fonction des intertextes implicites, voir Morris $2006: 279-286$, qui prend en examen les Mémoriaux de Klarsfeld et le rôle dérobé qu'ils jouent dans le roman.

\section{RÉSUMÉS}

L'article examine, à partir de la première édition de Dora Bruder de Patrick Modiano, la représentation de l'acte photographique, à travers neuf ekphrasis des portraits de la famille Bruder et de leur fille et de deux images énigmatiques d'un monde social et urbain disparu que l'auteur choisit d'évoquer. L'analyse, à travers les documents d'archives insérés dans la diégèse, se propose de montrer l'impuissance du déclic photographique devant la reconstruction de l'histoire de Dora. Les images en prose sont ensuite étudiées de façon à montrer comment l'écriture magnétise et réfracte le cliché, qui n'est pas reproduit dans le livre. La cohérence textuelle des descriptions des clichés au niveau de l'énoncé et de la signification dissimule des manques, des trous. L'absence de connotation émotionnelle réservée aux ekphrasis des portraits de famille apparaît comme un vide nécessaire au narrateur pour entrer en résonance avec la disparue.

The article analyses - starting from the first edition of Patrick Modiano's Dora Bruder-the representation of the photographic act, the nine ekphrasis of portraits of the Bruder's family and their daughter and the two puzzling pictures of a social and urban world which disappeared. The goal of the analysis is to show the impotence of the photographic object when faced with the reconstruction of Dora's story and to compare it to the official documents inserted in the diegesis. The analysis then considers the pictures in prose to show how writing magnetises and refracts the cliché, being this document absent in the narration. The textual coherence of these descriptions on the level of the discourse and of the significance conceals some gaps. The absence of emotional connotation - reserved to the ekphrasis of family portraits - appears like a gap necessary for the narrator to create a resonance, through narrative, with the disappeared girl.

\section{INDEX}

Index géographique : France

Index chronologique : XXe siècle

Mots-clés : cohérence textuelle, ekphrasis, mémoire, Occupation, oubli, photographie 


\section{AUTEUR}

\section{VALERIA SPERTI}

Université de la Basilicate 